\title{
Composición y estructura en agregaciones de moluscos terrestres en el Complejo de vegetación de mogote, Escaleras de Jaruco, Cuba
}

\author{
Maike Hernández Quinta ${ }^{1 *} \&$ Bernardo Reyes Tur ${ }^{2}$ \\ 1. Instituto de Ecología y Sistemática, La Habana; maike@ecologia.cu, Maike.hernandez@gmail.com \\ 2. Universidad de Oriente, Facultad de Ciencias Naturales, Ave. Patricio Lumumba, Santiago de Cuba 90500, Cuba; \\ breyes@cnt.uo.edu.cu \\ * Correspondencia
}

Recibido 13-IX-2012. Corregido 08-V-2013. Aceptado 04-VI-2013.

\begin{abstract}
Structure and composition of terrestrial molluscs assemblages on the mogote vegetation complex of Escaleras de Jaruco, Cuba. Cuba has one of the richest land snail faunas of the world. This important fact has promoted different kind of studies on this group to promote conservation programs, from which many studies have directed their efforts to inventories, and population and community ecology. To contribute with this population knowledge, we studied land snails assemblages in three karstic elevations at the "Escaleras de Jaruco-Tapaste-Cheche" Natural Protected Landscape, Mayabeque, Cuba. We aimed to analyze the variation of the composition and structure of the assemblages between the rainy and little rainy months. The study was conducted from August to November 2009 and from January to April, 2010, in ten permanent square plots $\left(9 \mathrm{~m}^{2}\right)$ separated for over 20m, on each elevation (Beluca, La Chirigota and La Jaula). In each plot, only live individuals were registered (physiologically active and at rest) to obtain species richness and abundance; besides, temperature $\left({ }^{\circ} \mathrm{C}\right)$ and relative humidity $(\%)$ were also considered in each plot. A total of 4248 individuals were observed which comprised two subclasses, five orders, 11 families, 20 genera and 21 species of terrestrial molluscs. From the total, 19 were Cuban endemics and eight were exclusive from Mayabeque, Matanzas. The Jaula showed the greater riches with 19 species, followed of Beluca with 17, and The Chirigota with 15 . In the rainy months, La Jaula, showed individual's greater abundance with 1707, followed of Beluca with 1305 and La Chirigota with 1236. We observed differences in the population density in the three elevations between the rainy and little rainy months, which can be due to the climatic adverse conditions that are shown at the little rainy months. Additionally, during the survey we observed dominance of prosobranch species over the pulmonates. The specific abundance curves showed a steep slope, although was major in the rainy months in relation to the little rain months, which indicates the presence of dominant, common and rare species in the assemblages. In this study, it was demonstrated than the riches and the abundance of land snails in all three elevations, was favored for the elevated relative humidity and the air temperature during the rainy months. These environmental conditions resulted fundamental for the survival of these terrestrial gastropods, as the reproduction and search of food were favored. Rev. Biol. Trop. 61 (4): 1769-1783. Epub 2013 December 01.
\end{abstract}

Key words: land snails, richness, abundance, prosobranchs, pulmonates, karstic forest, Cuba.

La fauna de moluscos terrestres y marinos registrada en Cuba es una de las más diversas del mundo, con más de 3000 especies, a pesar del origen relativamente reciente (en Pleistoceno aproximadamente) del archipiélago. En la fauna actual de moluscos terrestres cubanos, están bien representados los prosobranquios y pulmonados. Se han inventariado 1288 especies, lo que unido a las más de
2100 subespecies descritas (Espinosa \& Ortea, 2009), hacen de los gastrópodos terrestres un grupo distintivo entre los restantes invertebrados terrestres cubanos, con más de $96 \%$ de endemismo a nivel de especies.

Aunque existe una vasta bibliografía relacionada con los moluscos terrestres cubanos, el nivel de conocimiento es aún insuficiente y la información se encuentra dispersa y 
desactualizada desde el punto de vista taxonómico. Los estudios ecológicos antes de la década de 1980 consistían fundamentalmente en describir el hábitat que ocupaban las especies y la distribución geográfica (Henderson, 1916; Pérez, 1942; Herrera, 1945; Jaume, 1945, 1972). Por otra parte, en menor medida se han realizado estudios de comunidades (Bidart, Tadeo \& Alfonso, 1992; Bidart, Fernández, Osorio \& Reynaldo, 1996; Pérez, Villaseca \& Zione, 1996; Pérez, 1999; Oliva, 2004). El enfoque poblacional ha sido el más frecuente y ha estado referido principalmente a taxones carismáticos como Polymita spp. (Berovides, 1987; Bidart, Fernández \& Iglesias, 1998; Reyes-Tur, 2004), Caracolus sagemon Beck, 1837 (Pérez \& Ramos, 1997; Maceira, 2010), Liguus ssp. (Álvarez \& Berovides, 1989; Fernández \& Berovides, 2000) y Zachrysia guanensis Poey, 1857 (Tadeo \& Alfonso, 1997; Tadeo, Alfonso \& Berovides, 1999). Asimismo, las áreas protegidas han sido escenarios de varios estudios referentes a los moluscos terrestres (Jaume, 1972; Berovides, Tadeo \& Alfonso, 1994; Maceira, 2000; Maceira, 2005 a,b,c; Oliva-Oliveira \& Real, 2009). Sin embargo, en la actualidad para estas áreas, predominan los listados de especies y se desconoce sobre la composición estructural de las agregaciones de moluscos.

El Complejo de vegetación de mogote en el Paisaje Natural Protegido "Escaleras de Jaruco-Tapaste-Cheche", se caracteriza por una elevada abundancia de moluscos (González, 1989), y sólo se conoce una lista de especies. Por otra parte, se desconoce la estructura de las agregaciones de moluscos terrestres, por lo que el estudio pretende analizar cómo varía la composición y la estructura de las agregaciones entre los meses lluviosos y poco lluviosos.

\section{MATERIALES Y MÉTODOS}

Área de estudio: El trabajo se realizó en el Paisaje Natural Protegido "Escaleras de Jaruco-Tapaste-Cheche", provincia Mayabeque ubicado entre las coordenadas geográficas: $23^{\circ} 00^{\prime} 00^{\prime \prime} \mathrm{N}-82^{\circ} 01^{\prime} 27^{\prime}$ 'W y $23^{\circ} 03$ ' $27^{\prime \prime} \mathrm{N}$
- 8208’20” W. El área presenta un conjunto de elevaciones de estructura geosinclinal donde se produjo una inversión de relieve que dio lugar a un afloramiento cársico. Desde el punto de vista geomorfológico, las elevaciones son de naturaleza caliza del Mioceno, con $15 \mathrm{~km}$ de largo y entre 3 a $7 \mathrm{~km}$ de ancho, y se encuentran en un valle a $200 \mathrm{~m}$. s.n.m., con una altura máxima de 300m.s.n.m., posee embudos cársicos, carso cónico y de cúpulas; el tipo de suelo predominante es el cársico duro cavernoso (Cañas \& Ysalgué, 1978). El presente estudio se realizó en el complejo de vegetación de mogote, que se encuentra sobre las elevaciones cársicas. Esta formación se caracteriza por la presencia de bosques siempreverde y/o semideciduo, indistintamente según la exposición, el sustrato, las variantes del relieve y la altura de cada mogote. En la cima de las elevaciones se destacan matorrales con especies emergentes. En la mayoría de las elevaciones, la vegetación conserva sus elementos florísticos más representativos, su estructura y fisonomía (Oviedo et al., 2006).

Muestreo: Dentro del complejo de vegetación de mogote, se estudiaron tres elevaciones cársicas: Beluca $\left(23^{\circ} 01^{\prime} 31^{\prime \prime} \mathrm{N}-82^{\circ} 04^{\prime} 59^{\prime \prime}\right.$

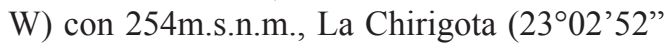
$\mathrm{N}-82^{\circ} 06^{\prime} 02^{\prime \prime}$ W) con 250m.s.n.m., y La Jaula $\left(23^{\circ} 00^{\prime} 59^{\prime \prime} \mathrm{N}-82^{\circ} 05^{\prime} 15^{\prime}\right.$ ' W) con 257 m.s.n.m. (Fig. 1). En cada elevación se seleccionaron al azar 10 parcelas de $9 \mathrm{~m}^{2}$ separadas unas de otras por más de $20 \mathrm{~m}$. Los muestreos se efectuaron mensualmente, cuatro meses del período lluvioso y cuatro meses del período poco lluvioso para un total de ocho meses en cada elevación. Los meses estudiados del período lluvioso fueron de agosto a noviembre 2009 y los meses del período poco lluvioso de enero a abril 2010. El esfuerzo de muestreo empleado en cada parcela fue de $40 \mathrm{~min} /$ recolector. Dentro de cada parcela se registraron solamente los individuos vivos (fisiológicamente activos y en reposo), el estudio fue de observación/trabajo de campo. En cada parcela se midió la temperatura $\left({ }^{\circ} \mathrm{C}\right)$ y la humedad relativa (\%) con un Higro-termómetro de marca EXTECH con un error de 


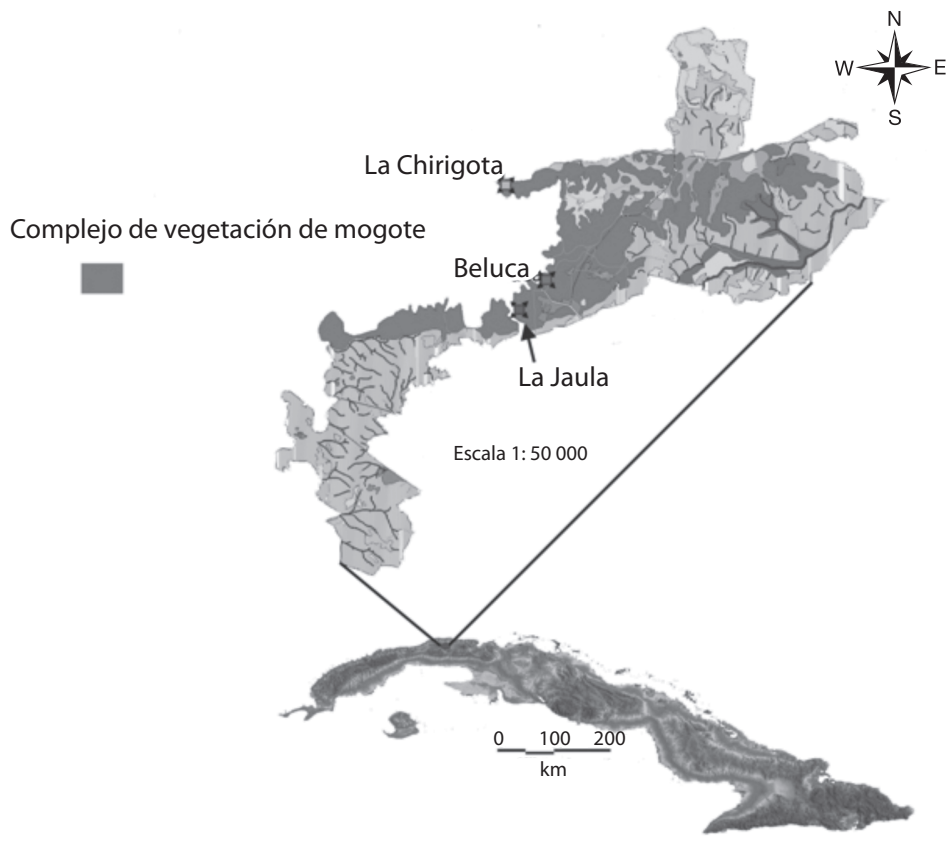

Fig. 1. Elevaciones cársicas en las "Escaleras de Jaruco-Tapaste-Cheche", Mayabeque, Cuba.

Fig. 1. The karstic elevations at the "Escaleras of Jaruco-Tapaste-Cheche" Natural Protected Landscape, Mayabeque, Cuba.

$0.1^{\circ} \mathrm{C}$ y $1 \%$, respectivamente, cada lectura de los datos se tomaron al finalizar cada parcela.

Identificación taxonómica: Fuera de las parcelas de trabajo se recolectó una muestra de referencia de cada taxón para facilitar su clasificación hasta el nivel específico. Para identificar los ejemplares fueron utilizados los catálogos de las familias Potamidae y Urocoptidae (Torre \& Bartsch, 1938, 1941, 2008). También se consultó la sección de moluscos terrestres de las colecciones zoológicas de la Academia de Ciencias de Cuba (CZACC) depositadas en el Instituto de Ecología y Sistemática. Se utilizó el ordenamiento taxonómico de Bouchet \& Rocroi (2005).

Análisis de la diversidad: Se empleó el programa EstimateS versión 7.5.2 (Colwell, 2005), para evaluar si el esfuerzo de muestreo produjo resultados representativos de las agregaciones y estimar la riqueza de los gastrópodos vivos. Se tuvieron en cuenta 80 muestras que provinieron de las 10 parcelas durante los ocho meses, en las tres elevaciones. Los estimadores no paramétricos seleccionados fueron ICE (Lee \& Chao, 1994), para conocer el número de especies esperadas en relación con las detectadas en diez o menos unidades de muestreo (Lee \& Chao, 1994). Chao2 (Chao, 1987) estima el número de las especies esperadas considerando la relación entre la cantidad de especies únicas (que aparecen en una muestra) y las duplicadas (que aparecen en dos muestras). Jackknife 1 (Burnham \& Overton, 1979) se basa en el número de las especies que están presentes solamente en una muestra, mientras que el estimador Bootstrap se basa en la proporción de unidades de muestreo que contienen a cada especie (Smith \& van Belle, 1984).

Para el análisis de la dominancia se construyeron curvas de rango-abundancia, una por elevación para cada período. Estas curvas representan el ordenamiento de las especies que conformaron las agregaciones de los moluscos terrestres, y se confeccionaron con los logaritmos decimales de la abundancia proporcional (Pi) de cada especie. 
El recambio de las especies o Diversidad $\beta$ entre las elevaciones y entre períodos climáticos fue calculado por el índice de similitud proporcional entre dos muestras (Hurlbert, 1978). Se realizó un análisis de Similitud SIMPER (siglas en inglés), en el cual se utilizaron como factores las elevaciones y los períodos, y las especies como las variables. Este procedimiento permitió conocer las especies que más contribuyeron a la similitud de las agregaciones entre ambos períodos. En este caso, se tuvieron en cuenta las especies cuyas contribuciones sumadas alcanzaran más de $80 \%$.

Para profundizar en la estructura de las agregaciones se comparó las abundancias de individuos operculados y pulmonados. En este sentido se comparó mediante un ANOVA de clasificación simple entre las tres elevaciones con el objetivo de ver si existía la misma tendencia de las abundancias de individuos operculados y pulmonados. Por último, se comparó variables micro-climáticas para ver si existían diferencias entre periodos, para lo cual se empleó una prueba $t$ de Student. El programa empleado fue STATISTICA versión 8.0. El $\alpha$ de rechazo utilizado fue de 0.05 .

\section{RESULTADOS}

Composición taxonómica: En las tres elevaciones cársicas de Escaleras de Jaruco, a partir de 4248 registros se identificaron 21 especies de moluscos terrestres incluidos en dos subclases, cinco órdenes, 11 familias, 20 géneros (Cuadro 1). Los prosobranquios, con 10 especies, estuvieron representados por las familias Potamiidae (5), Helicinidae (4) y Megalomastomatidae (1), mientras que los pulmonados con 11 especies se distribuyeron en ocho familias (Cuadro 1). De las especies detectadas, 19 son endémicas cubanas $(90 \%)$ y ocho de ellas son endémicas de la región Mayabaque-Matanzas (38\%), entre las que se encuentra Eutudora jimenoi un endémico local de las elevaciones cársicas de Tapaste-Jaruco (Cuadro 1).
Riqueza de especies y abundancia en las tres elevaciones cársicas: La Jaula presentó la mayor riqueza observada con 19 especies, seguida de Beluca con 17 y La Chirigota con 15. La Jaula mostró la mayor abundancia de individuos con 1 707, seguido de Beluca con 1305 y La Chirigota con 1 236. El Índice de Similitud Proporcional (SP) calculado entre las especies presentes en las tres elevaciones mostró mayor similitud entre Beluca y La Jaula (0.77) seguido de La Jaula y La Chirigota $(0.75)$, en tanto que la menor similitud de especies se reportó entre Beluca y La Chirigota (0.68).

De manera general, aunque hubo diferencias entre los distintos estimadores todos marcaron la misma tendencia en cada sitio y la estimación del total de especies fue similar, no obstante, solamente para La Jaula las curvas para todos los estimadores tienden a saturarse. En cambio para La Chirigota y Beluca no se aprecia saturación de estas curvas (Fig. 2).

Variación de la composición de especies entre períodos: El valor del índice de similitud proporcional entre los meses lluviosos y poco lluviosos, indicó mayor semejanza en la composición cuantitativa de las especies para La Chirigota ( $\mathrm{SP}=0.61$ ), seguido de Beluca $(\mathrm{SP}=0.53)$, y por último en La Jaula $(\mathrm{SP}=0.46)$. Los resultados obtenidos a partir del análisis SIMPER mostraron que en La Chirigota fueron cuatro especies las de mayor contribución a la similitud entre períodos climáticos, en la que se destaca Chondropoma pictum con $39 \%$. En tanto, en Beluca con sólo tres especies se alcanzó una contribución de casi $91 \%$ de similitud entre ambos períodos, nuevamente con un marcado aporte de C. pictum (53\%). Al igual que en Beluca, en La Jaula fueron tres las especies que más aportaron, y en este caso, Farcimen tortum fue la que mostró mayor contribución (38.6\%) (Cuadro 2).

Variación de la densidad, la temperatura y la humedad relativa entre meses lluviosos y poco lluviosos: En las tres localidades se registraron 531 individuos promedio para un 


\section{CUADRO 1}

Composición taxonómica de los moluscos terrestres registrados en las tres elevaciones cársicas muestreadas en el Paisaje Natural Protegido "Escaleras de Jaruco-Tapaste- Cheche, Mayabeque", Cuba

TABLE 1

Taxonomic composition of the land snails registered in the three karstic elevations sampled at the Natural Protected Landscape "Escaleras de Jaruco-Tapaste-Cheche, Mayabeque", Cuba

\begin{tabular}{|c|c|c|c|c|}
\hline \multirow{2}{*}{ Subclase Prosobrachia } & \multirow[t]{2}{*}{ Categorías taxonómicas } & \multicolumn{3}{|c|}{ Elevaciones } \\
\hline & & $\mathrm{J}$ & B & $\mathrm{CH}$ \\
\hline \multirow[t]{5}{*}{ Orden Cycloneritimorpha } & Familia Helicinidae & & & \\
\hline & Alcadia hispida (Pfeiffer, 1839) & $\mathrm{x}$ & $\mathrm{x}$ & \\
\hline & Helicina aspersa (Pfeiffer, 1839) & $\mathrm{x}$ & $\mathrm{x}$ & $\mathrm{x}$ \\
\hline & Ustronia sloanei (d'Orbigny, 1842) & $\mathrm{x}$ & $\mathrm{x}$ & $\mathrm{x}$ \\
\hline & Emoda submarginata (Gray, 1824) & & $\mathrm{x}$ & $\mathrm{x}$ \\
\hline \multirow[t]{2}{*}{ Orden Architaenioglossa } & Familia Megalomastomidae & & & \\
\hline & Farcimen tortum (Wood, 1828) & $\mathrm{x}$ & $\mathrm{x}$ & $\mathrm{x}$ \\
\hline \multirow[t]{6}{*}{ Orden Littorinimorpha } & Familia Potamiidae & & & \\
\hline & Rhytidopoma nodulatum (Poey, 1851) & $\mathrm{x}$ & $\mathrm{x}$ & $\mathrm{x}$ \\
\hline & Torrella immersa (Gundlach in Pfeiffer, 1857) & $\mathrm{x}$ & $\mathrm{x}$ & $\mathrm{x}$ \\
\hline & Chondropoma pictum (Pfeiffer, 1839) & $\mathrm{x}$ & $\mathrm{x}$ & $\mathrm{x}$ \\
\hline & Chondropoma auberianum (d'Orbigny, 1842) & $\mathrm{x}$ & $\mathrm{x}$ & \\
\hline & Eutudora jimenoi (Arango in Pfeiffer, 1864) & $\mathrm{x}$ & $\mathrm{x}$ & $\mathrm{x}$ \\
\hline \multicolumn{5}{|l|}{ Subclase Pulmonata } \\
\hline \multirow[t]{17}{*}{ Orden Stylommatophora } & Familia Urocoptidae & & & \\
\hline & Gongylostoma planospira (Pfeiffer, 1855) & $\mathrm{x}$ & $\mathrm{x}$ & $\mathrm{x}$ \\
\hline & Microceramus turricola (Pfeiffer, 1839) & & & $\mathrm{x}$ \\
\hline & Familia Orthalicidae & & & \\
\hline & Liguus fasciatus(Müller, 1774) & $\mathrm{x}$ & & $\mathrm{x}$ \\
\hline & Familia Oleacinidae & & & \\
\hline & Oleacina straminea (Deshayes, 1819) & $\mathrm{x}$ & $\mathrm{x}$ & $\mathrm{x}$ \\
\hline & Familia Sagdidae & & & \\
\hline & Hojeda boothiana (Pfeiffer, 1839) & $\mathrm{x}$ & $\mathrm{x}$ & \\
\hline & Setipellis stigmatica (Pfeiffer, 1841) & $\mathrm{x}$ & $\mathrm{x}$ & $\mathrm{x}$ \\
\hline & Familia Gastrodontidae & & & \\
\hline & Zonitoides arboreus (Say, 1816) & $\mathrm{x}$ & & \\
\hline & Familia Camaenidae & & & \\
\hline & Zachrysia auricoma (Férussac, 1822) & $\mathrm{x}$ & $\mathrm{x}$ & $\mathrm{x}$ \\
\hline & Familia Cepolidae & & & \\
\hline & Jeanneretia bicincta (Menke, 1830) & $\mathrm{x}$ & $\mathrm{x}$ & $\mathrm{x}$ \\
\hline & Eurycampta bonplandi (Lamarck, 1822) & $\mathrm{x}$ & & \\
\hline \multirow[t]{2}{*}{ Orden Systellommatophora } & FamiliaVeronicelidae & & & \\
\hline & Veronicella sp. & $\mathrm{x}$ & $\mathrm{x}$ & \\
\hline
\end{tabular}

Beluca: B, La Chirigota: CH, La Jaula: J. 

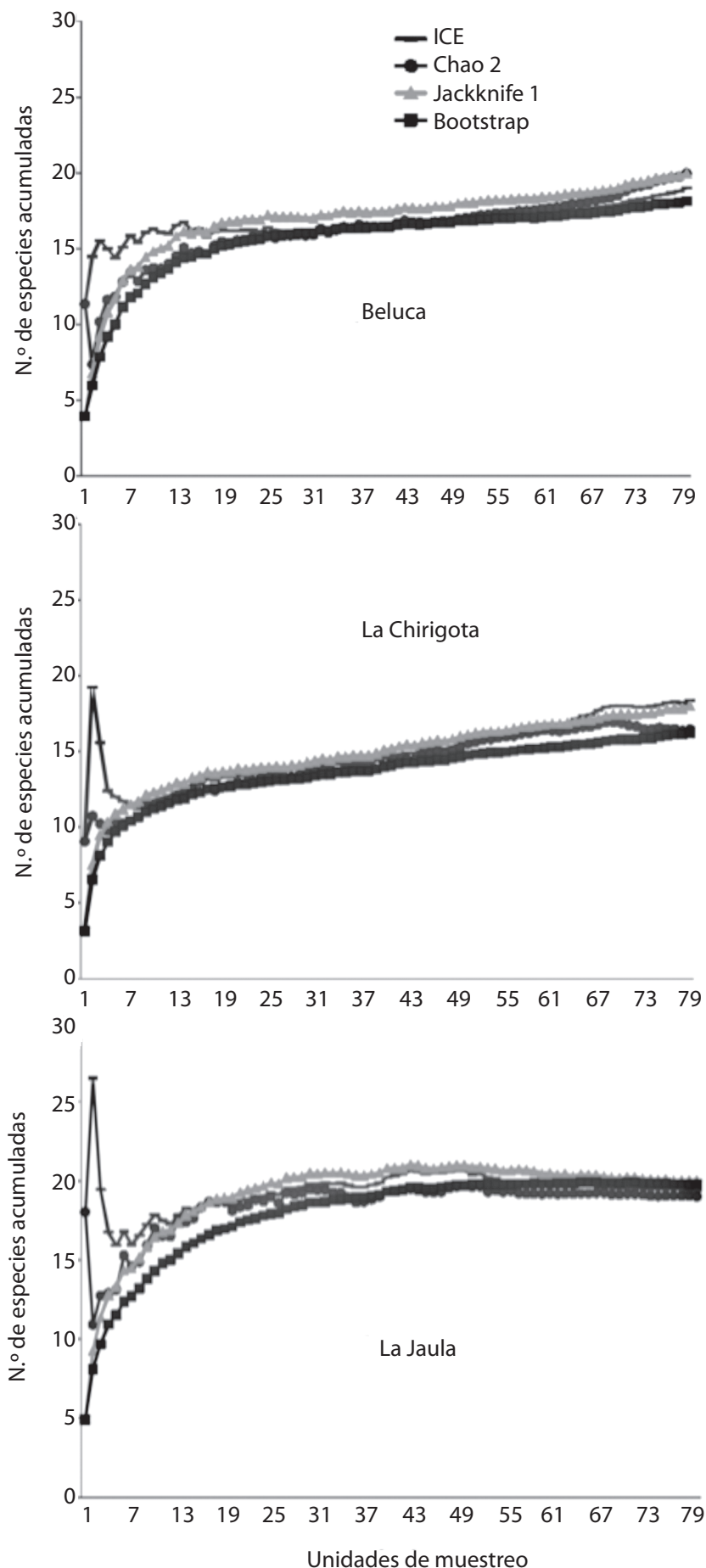

Fig. 2. Curvas de acumulación de especies de moluscos terrestres a partir de los estimadores ICE, Chao 2, Jackknife 1 y Boostrap, en las tres elevaciones cársicas de Escaleras de Jaruco, Cuba.

Fig. 2. Species accumulation curves of the land snails by means of the estimators ICE, Chao 2, Jackknife 1 and Boostrap, in the three karstic elevations of Escaleras de Jaruco, Mayabeque, Cuba. 
CUADRO 2

Abundancia y porcentajes de las similitudes de las especies de moluscos terrestres entre los períodos de meses lluviosos y poco lluviosos en Escaleras de Jaruco, Mayabeque, Cuba

TABLE 2

Abundance and similarity percentages of land snail species between the rainy and little rainy months in Escaleras de Jaruco, Mayabeque, Cuba

\begin{tabular}{lccccc}
\multicolumn{1}{c}{ Especies } & $\begin{array}{c}\text { Promedio } \\
\text { Abundancia }\end{array}$ & $\begin{array}{c}\text { Promedio } \\
\text { Similitud }\end{array}$ & $\begin{array}{c}\text { Desviación } \\
\text { estándar } \\
\text { de la Similitud }\end{array}$ & $\begin{array}{c}\text { Contribución } \\
(\%)\end{array}$ & $\begin{array}{c}\text { Contribución } \\
\text { acumulada (\%) }\end{array}$ \\
Chondropoma pictum & 3.02 & \multicolumn{2}{c}{ La Chirigota } \\
Farcimen tortum & 2.55 & 5.57 & 0.54 & 39.01 & 39.01 \\
Torrella inmersa & 7.23 & 2.91 & 0.28 & 20.41 & 59.42 \\
Ustronia sloanei & 1.52 & 2.63 & 0.34 & 18.46 & 77.88 \\
& & 0.92 & 0.3 & 6.43 & 84.31 \\
Chondropoma pictum & 4.02 & 11.03 & 0.7 & 53.19 & 53.19 \\
Farcimen tortum & 7.9 & 5.46 & 0.38 & 26.3 & 79.49 \\
Torrella inmersa & 4.12 & 2.38 & 0.43 & 11.48 & 90.97 \\
& & Beluca & & & 38.67 \\
Farcimen tortum & 7.23 & 6.18 & 0.41 & 38.67 & 65.93 \\
Chondropoma pictum & 4.32 & 4.35 & 0.4 & 27.26 & 82.76 \\
Torrella inmersa & 7.7 & 2.69 & 0.38 & 16.83 & \\
\hline
\end{tabular}

Fueron consideradas sólo las especies cuyas contribuciones sumadas llegaron hasta $80 \%$. Only the species whose summed contributions reached over $80 \%$, were considered.

área de $210 \mathrm{~m}^{2}$. Al relacionar gráficamente la densidad de las especies en los meses muestreados con los registros de la temperatura y de la humedad relativa, se observó que las agregaciones presentaron un comportamiento similar en las tres elevaciones. En general, disminuyó la densidad durante los meses poco lluviosos, incluso al incrementarse ambas variables climáticas en el mes de abril, la densidad se mantuvo baja. (Fig. 3).

En los meses lluviosos los valores de la temperatura oscilaron entre 22 y $26^{\circ} \mathrm{C}$ y la humedad relativa entre 80 y $92 \%$, mientras que en los meses poco lluviosos se registraron valores de temperatura entre 18 y $23^{\circ} \mathrm{C}$ y la humedad relativa entre 71 y $82 \%$. La prueba$\mathrm{t}$ aplicada para la comparación de las medias de la temperatura y de la humedad relativa entre los meses lluviosos y poco lluviosos mostró diferencias significativas $(\mathrm{p}<0.05)$ para las tres elevaciones estudiadas (Beluca: temperatura $\mathrm{t}=14.8$ y humedad relativa $\mathrm{t}=6.4$;
La Chirigota: temperatura $\mathrm{t}=21.8$ y humedad relativa $\mathrm{t}=12.2$; La Jaula: temperatura $\mathrm{t}=9.2$ y humedad relativa $\mathrm{t}=13.4$ ).

Dominancia: Durante todos los muestreos se registró dominancia de las especies operculadas sobre las pulmonadas. Estas últimas alcanzaron los mayores valores de abundancia proporcional entre noviembre y abril (Fig. 4 A). Esta tendencia resultó similar para todas las elevaciones, la cual fue más evidente en La Jaula (Fig. 4 B). Pese a que la abundancia de los prosobranquios en La Jaula fue superior a la de Beluca y La Chirigota, no se apreciaron diferencias significativasentre las elevaciones $(\mathrm{H}=0.74 ; \mathrm{p}=0.69 ; \mathrm{N}=24)$, e igualmente ocurrió para los pulmonados entre las tres elevaciones $(\mathrm{H}=4.0 ; \mathrm{p}=0.13 ; \mathrm{N}=24)$ (Fig. $4 \mathrm{~B})$.

Las curvas de rango-abundancia confeccionadas para las tres elevaciones tienen una pendiente pronunciada, aunque de manera general, en los meses lluviosos fue mayor 

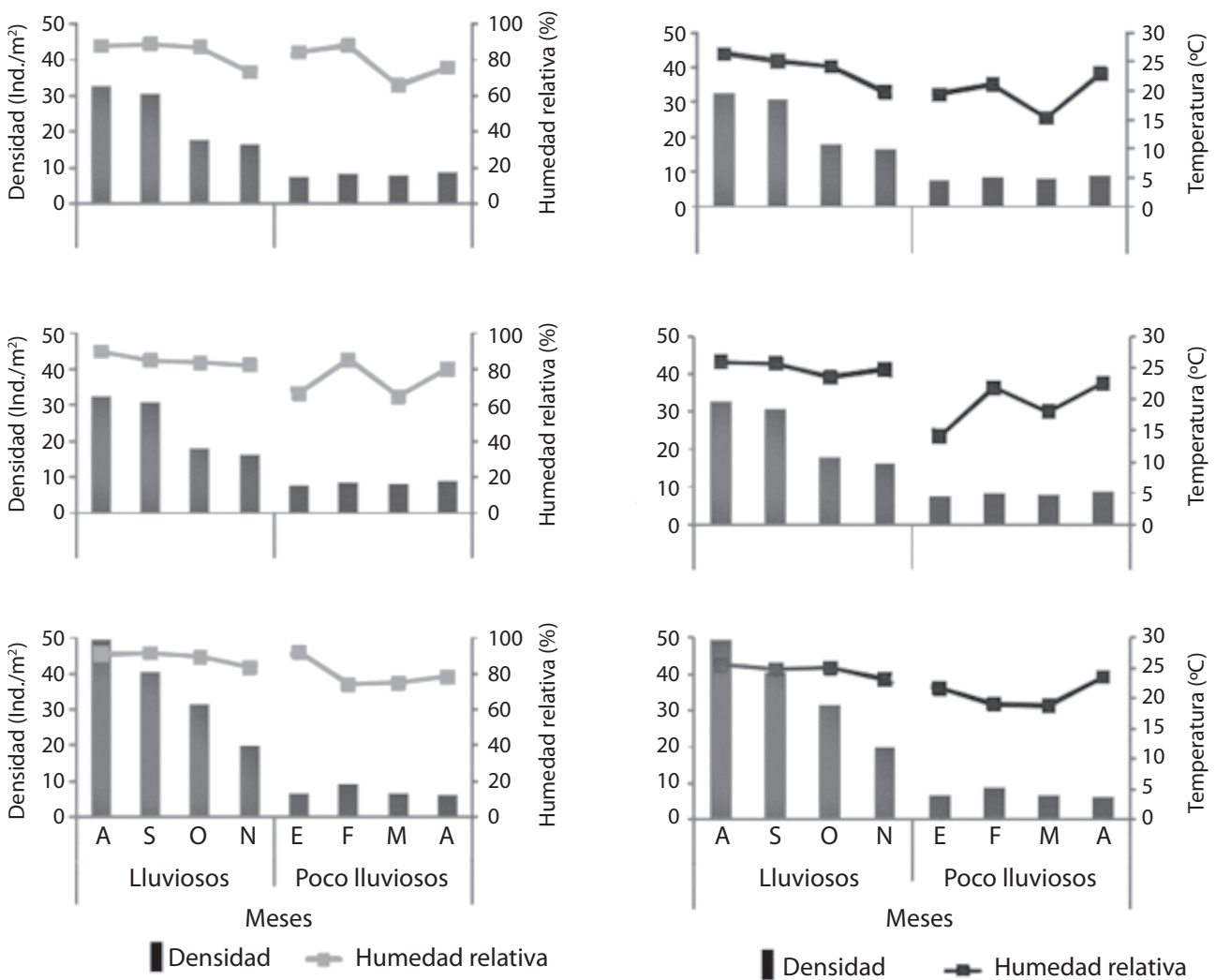

Fig. 3. Variación de la densidad poblacional de los moluscos terrestres, temperatura y humedad relativa en los meses lluviosos (agosto: A; septiembre: S; octubre: O; noviembre: N) y poco lluviosos (enero: E; febrero: F; marzo: M; abril: A), en tres elevaciones cársicas de Escaleras de Jaruco, Mayabeque, Cuba.

Fig. 3. Variation of land snails population densities, temperature and relative humidity between rainy months (August: A; September: S; October: O; November: N) and little rainy months (January: E; February: F; March: M; April: A), in the three karstic elevations at Escaleras de Jaruco, Mayabeque, Cuba.

respecto a los meses poco lluviosos, lo cual indica la presencia de especies dominantes, comunes y raras en las agregaciones. De manera general se aprecia que en las tres elevaciones en ambos periodos las especies dominantes son prosobranquios (Fig. 5).

Entre meses lluviosos y poco lluviosos, se comprobó variación en el orden de dominancia de algunas especies, entre las que se destacaron Ustronia sloanei y Rhytidopoma nodulatum, debido a que en las tres elevaciones y en ambos períodos variaron sus posiciones en las curvas. Otras especies mostraron pequeñas variaciones, entre estas se destacaron Farcimen tortum que durante los meses lluviosos y poco lluviosos en las tres elevaciones estuvo entre las especies con mayor dominancia (Fig. 5).

\section{DISCUSIÓN}

Composición y riqueza de las especies: Se registraron 21 especies de moluscos terrestres, de las cuales 17 coincidieron con las listadas para el área de estudio (González, 1989), que representan $59 \%$ respecto a la biocenosis del mogote de Escaleras de Jaruco detectada por este autor. De las cuatro especies restantes, Torrella inmersa, Microceramus turricola y 
A

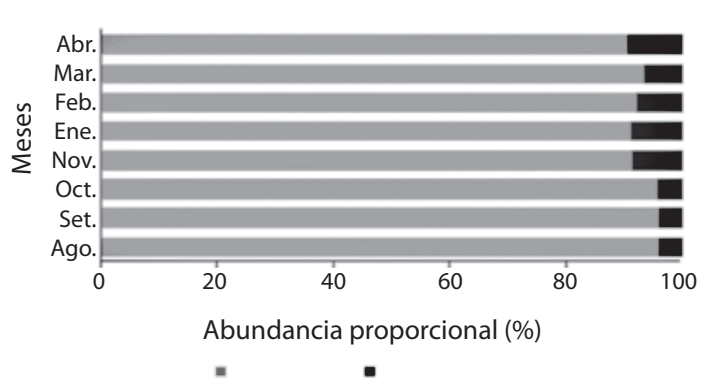

B

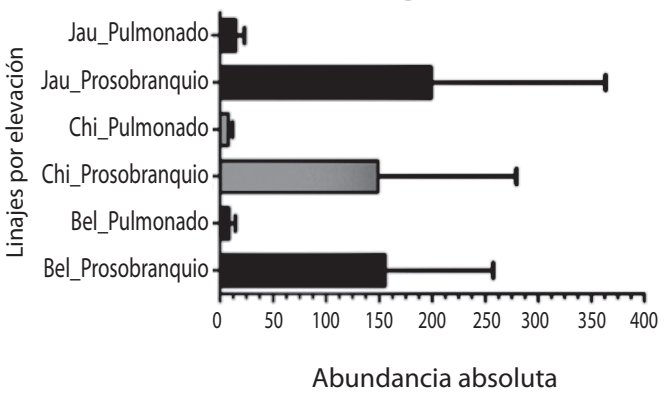

Fig. 4. A: Abundancia proporcional de moluscos terrestres prosobranquios y pulmonados en los meses lluviosos (agosto: Ago; septiembre: Sep; octubre: Oct; noviembre: Nov) y poco lluviosos (enero: Ene; febrero: Feb; marzo: Mar; abril: Abr), uniendo los datos de las tres elevaciones. B: Media y desviación estándar de la abundancia absoluta de los prosobranquios y los pulmonados en las tres elevaciones (Jau: La Jaula; Chi: La Chirigota; Bel: Beluca) en Escaleras de Jaruco, Mayabeque, Cuba.

Fig. 4. A: Proportional abundance of operculate and pulmonate land snails in rainy months (August: Ago; September: Sep; October: Oct; November: Nov) and little rainy months (January: Ene; February: Feb; March: Mar; April: Abr), joining the data of the three elevations. B: Mean and standard deviation of absolute abundance of operculate and pulmonate individuals in the three karstic elevations (Jau: La Jaula; Chi: La Chirigota; Bel: Beluca) in Escaleras de Jaruco, Mayabeque, Cuba.

Hojeda boothiana son nuevos registros para Escaleras de Jaruco. Por su parte, Emoda submarginata lo es para el complejo de vegetación de mogote. Uno de los rasgos que caracterizó la malacofauna de estas tres elevaciones fue su elevado endemismo; ocho de las especies registradas fueron endémicas de la región de Mayabeque-Matanzas. Lo anterior pudiera ser un reflejo del aislamiento geográfico que sufrió el área como consecuencia del origen de las alturas y del constante ascenso y descenso del nivel del mar entre el Eoceno Medio y el Pleistoceno (Iturralde-Vinent \& MacPhee, 1999).

De acuerdo con las curvas de acumulación de especies, se logró inventariar gran parte de la malacofauna presente en las tres elevaciones estudiadas, si se tiene en cuenta que los estimadores no paramétricos de diversidad utilizados son apropiados para muestras pequeñas (Colwell \& Coddington, 1994). Las curvas tienden a formar una asíntota, lo cual sugiere que si se incrementa el esfuerzo de muestreo en estas elevaciones, la probabilidad de encontrar nuevas especies de moluscos será baja. Aunque el esfuerzo de muestreo empleado en Beluca y La Chirigota con respecto a La Jaula no fue suficiente y debe aumentarse para encontrar una mayor riqueza de especies.
La riqueza detectada en las tres elevaciones fue baja, si se tiene en cuenta la elevada diversidad malacológica que se registra para la fauna cubana (Espinosa \& Ortea, 2009), lo que representa el 2\% de las 1288 especies descritas. Los valores de la riqueza más elevados, se han obtenido en inventarios realizados en otras regiones naturales del archipiélago cubano similares a la estudiada. Por ejemplo, en el Pan de Guajaibón se registraron 50 especies (Espinosa, Ortea, Oliva \& Milera, 2005); en Sierra Pan de Azúcar, 42 (Oliva, 2004); en el Yunque de Baracoa, 48; Sierra de Cubitas, 47 (Espinosa \& Ortea, 1999) y Lomas de Fomento, 29 especies (Pérez, Orozco \& Cañizares, 2010). No obstante, al comparar la riqueza de los moluscos detectados en el presente trabajo con las de otras localidades cubanas similares, la misma fue notablemente superior a la malacofauna de Sierra de Caballos (14 especies) y en Sierra de las Casas (14 especies) (Espinosa \& Ortea, 1999).

En comparación con otras localidades que presentan formaciones vegetales diferentes a la estudiada, como el Bosque Pluvial Montano, se aprecia que la riqueza registrada en este estudio fue superior, tales son los casos de los estudios realizados en La Bayamesa con 


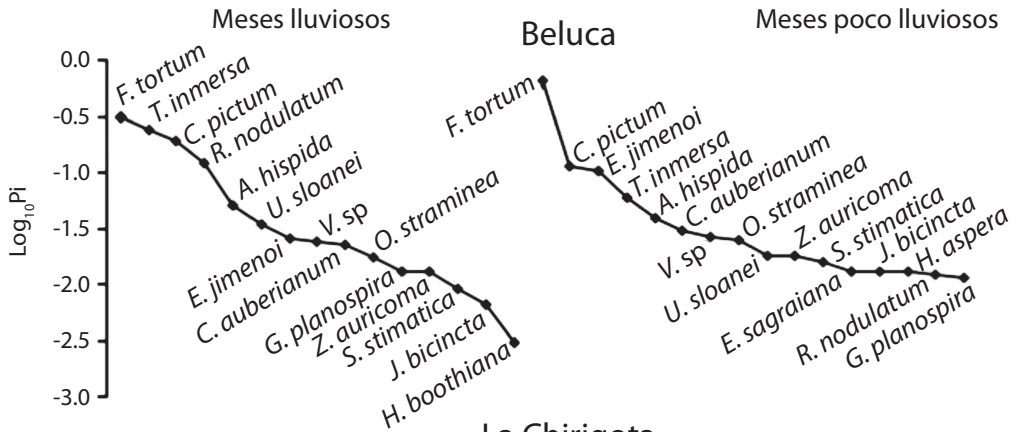

La Chirigota
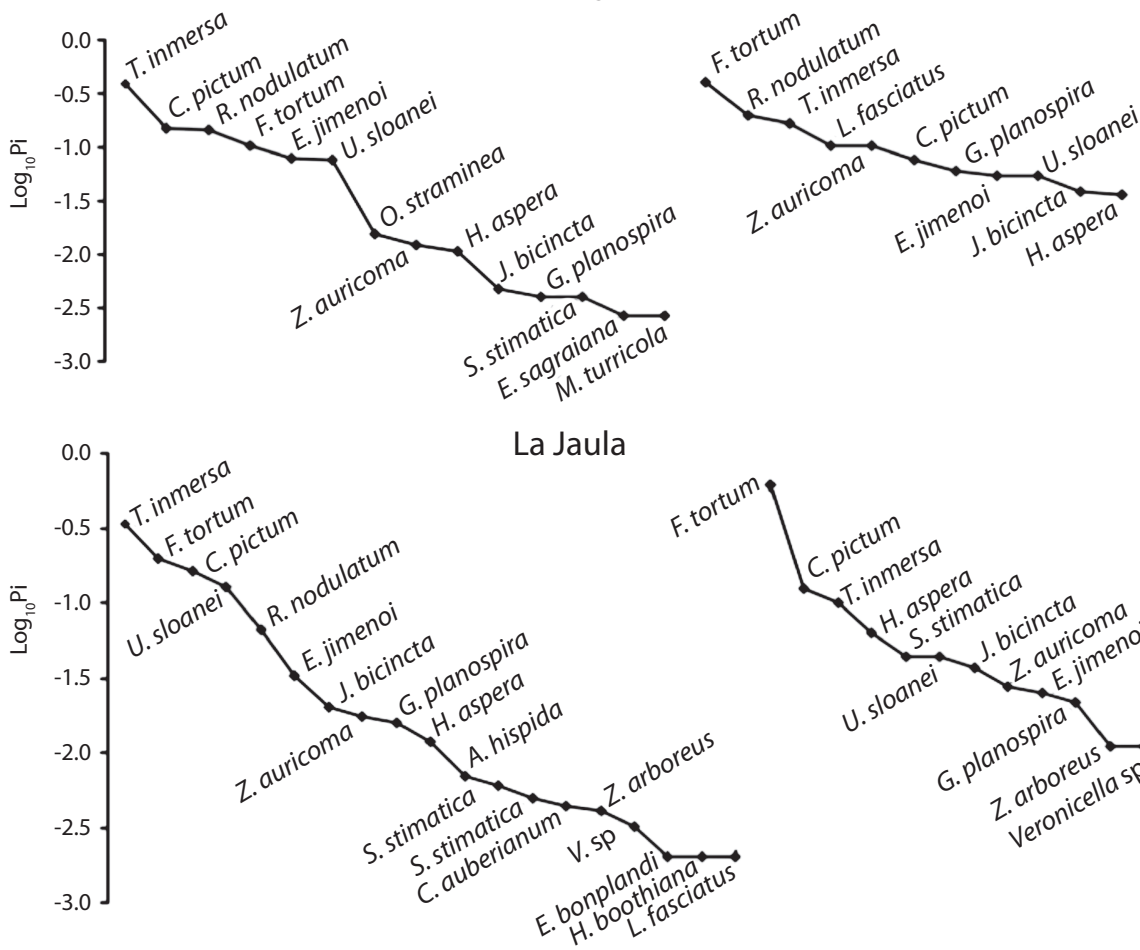

Especies

Fig. 5. Curvas de rango-abundancia (Pi es la abundancia proporcional) de las especies de moluscos terrestres presentes en las tres elevaciones cársicas (meses lluviosos y poco lluviosos) en Escaleras de Jaruco, Cuba.

Fig. 5. Rank abundance curves ( $\mathrm{Pi}$ is the proportional abundance) of the land snails species present in the three karstic elevations (rainy and little rainy months) in Escaleras de Jaruco, Cuba.

13 especies donde los moluscos pulmonados están mejor representados que los prosobranquios, Pico Turquino y Gran Piedra con 12 y 11 especies respectivamente (Maceira, 2005c). En la región de Alejandro de Humboldt en la cual aparecen zonas con Bosque Pluvial Montano y zonas con vegetación de mogote la fauna de moluscos es superior a la del área estudiada, donde los moluscos prosobranquios están mejor representados que los pulmonados (Maceira, 2005b).

Variación la densidad de especies en las elevaciones: En comparación a lo registrado 
por algunos autores, la densidad de individuos observada en el presente estudio fue baja (531 individuos promedio para un área de $210 \mathrm{~m}^{2}$ ). Por ejemplo, para un bosque de Madagascar se registraron 2430 individuos presentes en 0.04ha (Emberton, Pearce \& Randalana, 1999). En $1 \mathrm{~km}^{2}$ en un bosque de Camerún se encontraron 2654 individuos en 36 parcelas (De Winter \& Gittenberger, 1998). En un bosque de Nigeria, se detectaron 1442 individuos en $1 \mathrm{~km}^{2}$ (Oke \& Alohan, 2006). Sin embargo, en varios hábitats de Borneo, en 36 parcelas de $1 \mathrm{~km}^{2}$ se registraron 374 individuos (Schilthuizen \& Rutjes, 2001); menos de lo registrado en el presente estudio.

Solem (1973) y Kadmon \& Heller (1998) platearon la estrecha relación entre los moluscos terrestres y precipitaciones. Las diferencias encontradas en los valores de la densidad de las especies entre los meses lluviosos y poco lluviosos para las tres localidades estudiadas fueron significativas, lo cual coincide con lo planteado por los autores, ya que durante los meses lluviosos se crean condiciones propicias de alta humedad que favorecieron la actividad, la distribución y la diversidad de los gastrópodos terrestres (Berry, 1963; Russell-Hunter, 1964; Boag, 1985). Lo anterior coincide con lo hallado para los moluscos de otras regiones tropicales como son para el sur de Nuevo León (Correa-Sandoval, 1993, 1996-1997; MartínezRamírez, 2004) y San Luis de Potosí (CorreaSandoval, Strenth, Rodríguez \& Horta, 2009) ambas localidades ubicadas en México.

Es posible que durante los meses poco lluviosos, la disminución de la abundancia de los moluscos se deba en gran medida a que algunas de las especies pudieron estar afectadas por las condiciones climáticas como la baja humedad y temperatura y la disponibilidad de alimentos. En este sentido, las precipitaciones constituyeron una de las variables climáticas más importantes para el desarrollo de los moluscos, por ejemplo entre las especies que pudieran estar más afectadas aparecen Ustronia sloanei y Rhytidopoma nodulatum ya que en los meses poco lluviosos presentaron menor abundancia, sin embargo durante los meses lluviosos fueron especies dominantes. La disminución de la densidad poblacional de los moluscos terrestres causada por las condiciones adversas del clima, ha sido registrada para la subespecie cubana Liguus fasciatus achatinus (Fernández \& Berovides, 2000) y foráneas como Vitrina pelluscida y Euconulus fulvus en Polonia (Umiński \& Focht, 1979) y Vertigo geyeri en Wales, Reino Unido (Sharland, 2001).

Dominancia y abundancia de especies: Al comparar las tres elevaciones dentro de cada grupo de meses no se apreciaron grandes cambios en la estructura de las agregaciones, puesto que las relaciones de dominancia fueron similares. De manera general, coincidieron muchas de las especies dominantes, comunes y poco abundantes. En cambio, entre ambos períodos de meses se apreciaron grandes variaciones, sobre todo en el número de especies $\mathrm{y}$ en las relaciones de las dominancias entre las especies, además en los meses lluviosos prácticamente fueron cuatro las especies dominantes: Rhytidopoma nodulatum, Torrella inmersa, Farcimen tortum y Chondropoma pictum, mientras que en los meses poco lluviosos Farcimen tortum fue la única dominante. Estas diferencias parecen estar relacionadas, fundamentalmente, con las condiciones de la temperatura y la humedad creadas en los períodos de meses estudiados.

Farcimen tortum, encontrada esencialmente en el suelo, fue muy abundante en los meses lluviosos, aunque los valores más elevados los alcanzó en los meses poco lluviosos. Este resultado puede estar relacionado con el incremento de la hojarasca, y el consecuente aumento de las condiciones favorables de humedad y disponibilidad de alimentos durante dicho período. Teniendo en cuenta que la alimentación de esta especie se sustenta básicamente en el micelio de los hongos (Espinosa \& Ortea, 2009).

En este estudio se registraron tanto representantes de moluscos terrestres prosobranquios como pulmonados, y aunque estos últimos constituyen el grupo más numeroso del archipiélago cubano (Espinosa \& Ortea, 2009), en el área de estudio ambos estuvieron muy 
bien representados. Los miembros de Potamiidae se caracterizaron por presentar una mayor cantidad de especies, y entre las familias de prosobranquios cubanos es esta la más numerosa con 364 especies y la segunda en cuanto al número de especies entre pulmonados y prosobranquios (Espinosa \& Ortea, 2009).

Al parecer, las condiciones ambientales no afectaron la dominancia de los prosobranquios, ya que durante los meses lluviosos y poco lluviosos de ambas épocas predominaron sobre los pulmonados, aunque la abundancia de los prosobranquios fue ligeramente menor de noviembre a abril. La dominancia de los moluscos prosobranquios sobre las restantes especies pulmonadas ha sido documentada en varias zonas con afloramientos cársicos (Pérez, 1999; Oliva, 2004; Schilthuizen, Liew, Elahan \& Lackman-Ancrenaz, 2005). Oliva (2004) encontró mayor riqueza de pulmonados en Sierra Pan de Azúcar; sin embargo, en su estudio las abundancias más elevadas se registraron para los prosobranquios.

Poco se conoce de las adaptaciones funcionales de los gastrópodos prosobranquios terrestres, aunque se asemejan mucho a los pulmonados. Russell-Hunter (1964) planteó que prácticamente se desconoce la diapausa de las especies, aunque consideró al opérculo como una pre-adaptación que permite sellar la abertura de la concha y realizar estivaciones de corta duración durante las condiciones adversas, como ocurre en algunos annuláridos. Quizás esta adaptación permitió que las especies prosobranquias observadas en los meses lluviosos y poco lluviosos, mantuvieran elevada abundancia durante todo el período de estudio, aunque este pudiera ser el patrón entre épocas que sigan las especies operculadas de estas agregaciones bajo condiciones estables del clima. Además, es necesario conocer la duración del ciclo de vida, la tasa de reproducción, el tamaño de las puestas y la supervivencia de los estadios inmaduros, así como el tiempo de maduración de las estructuras reproductivas, ya que pueden ser factores relacionados con la abundancia de los prosobranquios de estas agregaciones.
La mayoría de las especies operculadas que fueron registradas en el carso, (Chondropomapictum, Torrella inmersa, Ustronia sloanei, Rhytidopoma nodulatum y Farcimentortum) o en el suelo (Farcimentortum), además de ser las más abundantes, con frecuencia se encontraron agrupadas, conducta que probablemente facilita su reproducción debido a la carencia de barreras físicas. En cambio los prosobranquios arborícolas (Helicina aspersa, C. auberianum), se encontraron más aislados y fueron menos abundantes, lo cual dificulta su reproducción y quizás esta sea una de las causas de la baja abundancia observada para estas especies en las tres elevaciones.

Una de las posibles razones de que los prosobranquios fueran tan abundantes y que se encontraran agrupados, es la presencia de sexos separados, lo cual aumentaría la probabilidad de la cópula entre machos y hembras; a diferencia de los moluscos pulmonados que son hermafroditas. Vernon (1995) planteó, que la ausencia de facilitación social (presencia de con-específicos) puede jugar un papel en la reducción del rendimiento reproductivo en los moluscos aislados, puesto que el apareamiento suele ser energéticamente muy costoso. Lo planteado por este autor pudiera reafirmar lo encontrado en nuestro estudio.

Con este trabajo se incrementa el conocimiento que existe de la estructura de las agregaciones de moluscos terrestres en el Paisaje Natural Protegido "Escaleras de JarucoTapaste-Cheche", por ello es vital incluir estos resultados en la preparación de los Planes y Estrategias de Conservación para esta área protegida dada la importancia de los moluscos terrestres en estos sitios. Se sugiere disminuir la explotación de los recursos naturales en el complejo de vegetación de mogote, ya que la mayoría de las especies de moluscos son exclusivas de esta formación vegetal.

\section{AGRADECIMIENTOS}

$\mathrm{Al}$ personal del área protegida de Escaleras de Jaruco, por permitir realizar la investigación en el área. A Betyna Neiyra, Annery Serrano, 
Anay Serrano, Ormaily Madruga y Rayner Núñez por el apoyo en el trabajo de campo. A Ileana Fernández y Grisel Cabrera por la lectura crítica y los valiosos comentarios y sugerencias realizadas. Este trabajo fue financiado por el proyecto "Creación, Conservación y Manejo de Colecciones Zoológicas" del Instituto de Ecología y Sistemática.

\section{RESUMEN}

Estudiamos las agregaciones de moluscos terrestres de tres elevaciones cársicas en el Paisaje Natural Protegido "Escaleras de Jaruco-Tapaste-Cheche", Mayabeque, Cuba. Se analizaron las variaciones de la composición y la estructura de las agregaciones entre los meses lluviosos y poco lluviosos. El estudio fue realizado desde agosto a noviembre 2009 y entre enero y abril 2010 en diez parcelas cuadradas permanentes $\left(9 \mathrm{~m}^{2}\right)$ en cada elevación. En cada parcela se registraron solamente los individuos vivos (fisiológicamente activos y en reposo), el estudio fue de observación/trabajo de campo. Dentro de cada parcela se registró la riqueza de especie y abundancia. En cada parcela se midió la temperatura $\left({ }^{\circ} \mathrm{C}\right)$ y la humedad relativa $(\%)$, se observaron 4248 individuos de dos subclases, cinco órdenes, 11 familias, 20 generos y 21 especies de moluscos terrestres; 19 son endémicos cubanos y ocho de ellos lo son de la región Mayabeque-Matanzas. Se observaron diferencias entre los meses lluviosos y poco lluviosos en la densidad poblacional entre las tres elevaciones, lo cual puede deberse a las condiciones climáticas adversas en los meses poco lluviosos. Durante los meses muestreados se detectó una dominancia de las especies operculadas sobre las pulmonadas. Las curvas de rango-abundancia se caracterizaron por tener una pendiente pronunciada, lo cual indica la presencia de especies dominantes, comunes $\mathrm{y}$ raras en las agregaciones.

Palabras clave: moluscos terrestres, riqueza, abundancia, prosobranquios, pulmonados, bosques cársicos, Cuba.

\section{REFERENCIAS}

Álvarez, J., \& Berovides, V. (1989). Ecología y polimorfismo en Liguus fasciatus (Mollusca: Pulmonata) de Cayo Romano. Revista Biología, 3, 183-196.

Berovides, V., Tadeo, R., \& Alfonso, M. (1994). Concluye el estudio sobre la malacocenosis de la Sierra de los Órganos, Pinar del Río. Cocuyo, 1, 7-8.

Berovides, V. (1987). Genética ecológica de Polymita picta roseolimbata Torre 1950, en Maisí. (Tesis doctoral). Universidad de la Habana, La Habana.
Berry, A. J. (1963). Growth and variation of the shell in certain Malayan limestone hill snails. Proceedings of the Malacological Society, 35, 203-206.

Bidart, L., Tadeo, R., \& Alfonso, C. (1992). Nicho ecológico de Polymita muscarum, Liguus fasciatus achatinus y Hermitrochus lucipeta. Ciencias Biológicas, 25, 9-19.

Bidart, L., Fernández, J., Osorio, M., \& Reynaldo, E. (1996). Moluscos terrestres del Yayal, provincia de Holguín. Cocuyo, 5, 25-26.

Bidart, L., Fernández, A., \& Iglesias, C. (1998). Reproducción de Polymita muscarum y Polymita venusta. Revista de Biología Tropical, 46, 683-689.

Boag, D. A. (1985). Microdistribution of three genera of small terrestrial snails (Stylommatophora: Pulmonata). Canadian Journal Zoology, 63(5), 1089-1095.

Bouchet, P. J., \& Rocroi, P. (2005) Classification and Nomenclator of Gastropod Families. Malacologia, 47, 1-397.

Burnham, K. P., \& Overton, W. S. (1979). Robust estimation of population size when capture probabilities vary among animals. Ecology, 60, 927-936.

Cañas, P., \& Ysalgué, S. (1978). Mapas de naturaleza y recursos: Geomorfología. In: C. Ibarra, M, Vegas, \& R. Espinosa (Eds.), Atlas de Cuba (pp. 30-31). La Habana: Instituto Cubano de Geodesia y Cartografía.

Chao, A. (1987). Estimating the population size for capture-recapture data with unequal catchability. Biometrics, 43, 783-791.

Colwell, R. K. (2005). EstimateS: Statistical estimation of species richness and shared species from samples. Versión 7.5. Retrieved from http://purl.oclc.org/ estimates.

Colwell, R. K., \& Coddington, I. A. (1994). Estimating terrestrial biodiversity through extrapolation. Philosophical Transactions of the Royal Society, 345, 101-118.

Correa-Sandoval, A., Strenth, N. E., Rodríguez, R., \& Horta, J. V. (2009). Análisis ecológico básico de los gastrópodos terrestres de la Región Oriental de San Luis Potosí, México. Acta Zoológica Mexicana, 25, 105-122.

Correa-Sandoval, A. (1993). Caracoles terrestres (Mollusca: Gastropoda) de Santiago, Nuevo León, México. Revista de Biología Tropical, 41, 683-687.

Correa-Sandoval, A. (1996-1997). Caracoles terrestres (Mollusca:Gastropoda) de Iturbide, Nuevo León, México. Revista de Biología Tropical, 44/45, 137-142.

De Winter, A. J., \& Gittenberger, E. (1998). The land snail fauna of a square kilometer patch of rainforest in southwestern Cameroon: high species richness, low 
abundance and seasonal fluctuations. Malacologia, 40, 231-250.

Emberton, K. C., Pearce, A., \& Randalana, R. (1999). Molluscan diversity in the unconserved Vohimena and the conserved Anosy mountain chains, Southeast Madagascar. Biological Conservation, 89, 183-188.

Espinosa, J., Ortea, J., Oliva, W., \& Milera, J. F. (2005). Moluscos terrestres y fluviales del Pan de Guajaibón, Área Protegida Mil Cumbres, Pinar del Río, Cuba. Revista Canarias de Ciencias, 16, 279-315.

Espinosa, J., \& Ortea, J. (1999). Moluscos terrestres del archipiélago cubano. Avicennia 2, 1-137.

Espinosa, J., \& Ortea J. (2009). Moluscos terrestres de Cuba.Vaasa, Finland: UCP Print.

Fernández, A., \& Berovides, V. (2000). Cambios de densidad de Liguus fasciatus achatinus en el Yayal, Holguín, Cuba. Revista Biología, 14, 141-146.

González, O. (1989). La biocenosis de las Escaleras de Jaruco y áreas cercanas. La Habana Cuba: Editorial Academia.

Henderson, I. B. (1916). A list of the land and fresh water shell of the Isle of Pines. Annals of the

Carnegie Museum, 10, 315-324.

Herrera, E. (1945). Moluscos colectados en la Julia, Zulueta. Revista de la Sociedad Malacologica Carlos de la Torre, 3, 1-18.

Hurlbert, S. H. (1978). The measurement of niche overlap and some relatives. Ecology, 59, 67-77.

Iturralde-Vinent, M., \& MacPhee, R. (1999). Paleogeography of the Caribbean region: Implications for Cenozoic biogeography. Bulletin American Museum Natural History, 238, 1-95.

Jaume, M. L. (1945). Excursión malacológica al Pan de Guajaibón. Revista de la Sociedad Malacologica Carlos de la Torre, 3, 73-83.

Jaume, M. L. (1972). Lista de los moluscos de la Sierra del Rosario. La Habana. Serie Biológica, 41, 1-29.

Kadmon, R., \& Heller, J. (1998). Modelling faunal response to climatic gradients with GIS: land snails as a case study. Journal Biogeography, 25, 527-539.

Lee, S. M., \& Chao, A. (1994). Estimating population size via sample coverage for closed capture-recapture models. Biometrics, 50, 88-97.

Maceira, D. (2000). Moluscos en Sierra Maestra. In N. Viña, G. A. Fong \& F. D. Maceira (Eds.), Diversidad biológica de los macizos montañosos de Sierra Maestra. Tomo 2. (pp. 607-643). Santiago de Cuba: Centro Oriental de Ecosistemas y Biodiversidad.

Maceira, D. (2005a). Moluscos terrestres. In D. Maceira, A. Fong, W. S. Alverson \& J. M. Shopland. (Eds.), Cuba: Siboney-Jutici. Rapid Biological Inventories. Report, 10. (pp. 54-55). Chicago: The Field Museum.
Maceira, D. (2005b). Terrestrial Mollusks. In D. Maceira, A. Fong, W. S. Alverson \& J. M. Shopland (Eds.), Cuba: Parque Nacional Alejandro de Humboldt. Rapid Biological Inventories. Report, 14. (pp. 184186). Chicago: The Field Museum.

Maceira, D. (2005c). Terrestrial Mollusks. In D. Maceira, A. Fong, W. S. Alverson \& J. M. Shopland (Eds.), Cuba: Parque Nacional La Bayamesa. Rapid Biological Inventories. Report, 13. (pp. 60-62). Chicago: The Field Museum.

Maceira, D. (2010). Dinámica poblacional de Caracolus sagemon en la Reserva Ecológica Siboney-Juticí, Santiago de Cuba. (Tesis doctoral). BIOECO, Santiago de Cuba, Cuba.

Martínez-Ramírez, V. H. (2004). Sinecología de los gastrópodos terrestres del Sur de Nuevo León, México. (Tesis Maestría). Instituto Tecnológico de Ciudad Victoria, Ciudad Victoria, México.

Oke, O. C., \& Alohan, F. I. (2006).The land snail diversity in a square kilometre of tropical rainforest in Okomu National Park, Edo State, Nigeria. African Scientist, 7, 135-142.

Oliva, W. (2004). Variación en las comunidades de moluscos terrestres de la Sierra Pan de Azúcar, Viñales. (Tesis de Maestría). Instituto de Ecología y Sistemática, La Habana, Cuba.

Oliva-Olivera, W., \& Real, R. (2009). Moluscos Terrestres de las Elevaciones Cársticas de Viñales, Pinar del Río, Cuba. Revista de Biología Tropical, 57, 589-604.

Oviedo, R. P., Ventosa, I. R., Vale, A. G., Loriga, J. P., Núñez, R. A., Rodríguez, A. G., \& Rodríguez, A. (2006). Elementos generales de la naturaleza en el Área Protegida Escalera de Jaruco-Loma el Cheche. Informe para la Empresa Nacional para la Protección de la Flora y la Fauna.

Pérez, A. M., Villaseca, J. C., \& Zione, N. (1996). Sinecología básica de moluscos terrestres en cuatro formaciones vegetales de Cuba. Revista de Biología Tropical, 44, 133-146.

Pérez, A. M., \& Ramos, I. (1997). Morfometría de tres poblaciones de Caracolus sagemon en Cuba. Revista de Biología Tropical, 45, 1563-1577.

Pérez, B. (1999). Composición y estructura ecológica de las malacocenosis en tres áreas cársicas de Sancti Spíritus. (Tesis de Maestría). Instituto de Ecología y Sistemática, La Habana, Cuba.

Pérez, B., Orozco, A., \& Cañizares, M. (2010). Los Moluscos terrestres de la Reserva Florística

Manejada "Lomas de Fomento" Sancti Spíritus. Cuba. Revista Infociencia, 14, 1-12.

Pérez, F. (1942). Moluscos de la región de Camoa y Somorrostro y sus condiciones de vida. Memorias de la Sociedad Cubana de Historia Natural Felipe Poey, $15,45-46$. 
Reyes-Tur, B. (2004). Ecología y Biología reproductiva de Polymita venusta (Gmelin, 1792). (Tesis doctoral). Universidad de Oriente, Santiago de Cuba, Cuba.

Russell-Hunter, W. (1964). Physiological aspects of ecology in nonmarine mollusks. In K. M. Wilbur, \& C. M. Yong (Eds.), Physiology of Mollusca, Volumen 1. (pp. 59-82). New York: Academic Press.

Schilthuizen, M., \& Rutjes, H. A. (2001). Land snail diversity in a square kilometre of tropical rainforest in Sabah, Malaysian Borneo. Journal of Molluscan Studies, 67, 417-423.

Schilthuizen, M., Liew, T. S., Elahan, B., \& LackmanAncrenaz, I. (2005). Effects of karst forest degradation on Pulmonate and Prosobranch land snail communities in Sabah, Malaysian Borneo. Conservation Biology, 19, 949-954.

Sharland, E. C. (2001). Autecology of Vertigo angustior and Vertigo geyeri in Wales. (Doctoral thesis). University of Sheffield, United Kingdom.

Smith, E. P., \& van Belle, G. (1984). Nonparametric estimation of species richness. Biometrics, 40, 119-129.

Solem, A. (1973). Island size and species diversity in Pacific island land snails. Malacologia, 14, 397-400.
Tadeo, R., \& Alfonso, M. (1997). Dispersión de Zachrysia guanensis costulata de la Sierra de San Carlos, Pinar del Río, Cuba. Revista Biología, 11, 141-144.

Tadeo, R., Alfonso, M., \& Berovides, V. (1999). Ecología de Zachrysia guanensis costulata de Sierra de San Carlos, Pinar del Río, Cuba. Revista Biología, 13, 95-102.

Torre, C. de la, \& Bartsch, P. (1938). The Cuban operculate land shells of the subfamily Chondropominae. Proceedings of the U.S. National Museum, 85, 193-425.

Torre, C. de la, \& Bartsch, P. (1941). The Cuban operculate land mollusks of the family Annulariidae. Proceedings of the U.S. National Museum, 89, 131-385.

Torre, C. de la, \& Bartsch, P. (2008). Los moluscos terrestres cubanos de la familia Urocoptidae. La Habana: Científico-Técnica.

Umiński, T., \& Focht, U. (1979). Population dynamics of some land gastropods in a forest habitat in Poland. Malacologia, 18, 181-184.

Vernon, J. G. (1995). Low reproductive output of isolated, self-fertilizing snails: inbreeding depression or absence of social facilitation? Proceedings the Royal Society London, 259, 131-136. 
